\title{
Thinking about a limited future enhances the positivity of younger and older adults' recall: Support for socioemotional selectivity theory
}

\author{
Sarah J. Barber ${ }^{1}$ - Philipp C. Opitz ${ }^{2}$ - Bruna Martins ${ }^{3}$. \\ Michiko Sakaki $^{4,5} \cdot$ Mara Mather $^{3}$
}

Published online: 25 April 2016

(C) Psychonomic Society, Inc. 2016

\begin{abstract}
Compared with younger adults, older adults have a relative preference to attend to and remember positive over negative information. This is known as the "positivity effect, " and researchers have typically evoked socioemotional selectivity theory to explain it. According to socioemotional selectivity theory, as people get older they begin to perceive their time left in life as more limited. These reduced time horizons prompt older adults to prioritize achieving emotional gratification and thus exhibit increased positivity in attention and recall. Although this is the most commonly cited explanation of the positivity effect, there is currently a lack of clear experimental evidence demonstrating a link between time horizons and positivity. The goal of the current research was to address this issue. In two separate experiments, we asked participants to complete a writing activity, which directed them to think of time as being either limited or expansive (Experiments 1 and 2) or did not orient them to think about time in a particular
\end{abstract}

Electronic supplementary material The online version of this article (doi:10.3758/s13421-016-0612-0) contains supplementary material, which is available to authorized users.

Sarah J. Barber

barber@sfsu.edu

1 Department of Psychology, San Francisco State University, 1600 Holloway Avenue, San Francisco, CA 94132, USA

2 Leonard Davis School of Gerontology, University of Southern California, Los Angeles, CA, USA

3 Department of Psychology, University of Southern California, Los Angeles, CA, USA

4 School of Psychology and Clinical Language Sciences, University of Reading, Reading, UK

5 Kochi University of Technology, Kami, Japan manner (Experiment 2). Participants were then shown a series of emotional pictures, which they subsequently tried to recall. Results from both studies showed that regardless of chronological age, thinking about a limited future enhanced the relative positivity of participants' recall. Furthermore, the results of Experiment 2 showed that this effect was not driven by changes in mood. Thus, the fact that older adults' recall is typically more positive than younger adults' recall may index naturally shifting time horizons and goals with age.

Keywords Aging $\cdot$ Positivity effect $\cdot$ Socioemotional selectivity theory $\cdot$ Memory $\cdot$ Emotion $\cdot$ Time perception

As people get older, they tend to show a relative preference to attend to and remember positive over negative information (Mather \& Carstensen, 2005). For example, relative to younger adults, older adults preferentially direct their eye gaze towards positive images, and/or away from negative images (Isaacowitz, Allard, Murphy, \& Schlangel, 2009; Isaacowitz, Wadlinger, Goren, \& Wilson, 2006a, 2006b; Knight et al., 2007). Similarly, relative to younger adults, older adults usually recall and recognize more positive and fewer negative images (Charles, Mather, \& Carstensen, 2003; Mather \& Knight, 2005), remember more positive autobiographical events (Kennedy, Mather, \& Carstensen, 2004; Levine \& Bluck, 1997), and remember their decisions as being associated with more positive outcomes (Mather \& Johnson, 2000). Together, this age-by-valence interaction is known as the positivity effect (Kennedy et al., 2004). Although some studies have failed to observe the positivity effect (e.g., Gruhn, Smith, \& Baltes, 2005; Kensinger, Brierley, Medford, Growdon, \& Corkin, 2002), a recent meta-analysis of 100 empirical studies confirmed that such age-related positivity effects are reliable, and increase in 
magnitude as the age gap between younger and older adults increases (Reed, Chan, \& Mikels, 2014).

Although the age-related positivity effect is a robust finding, there is still debate about why it occurs. The most widely cited explanation is based upon socioemotional selectivity theory (see Mather \& Carstensen, 2005; Reed \& Carstensen, 2012). According to socioemotional selectivity theory (Carstensen, 1993, 1995, 2006), people have a core constellation of social goals that operate across the lifespan. For example, people are generally motivated to acquire new knowledge about the world and to satisfy their emotional needs. However, the relative salience of specific goals fluctuates as a function of perceptions of time and life expectancy.

In general, younger adults perceive time as being expansive. This causes them to prioritize future-oriented goals, such as acquiring new knowledge and expanding their social networks, as these activities will help them prepare for the uncertain future challenges ahead of them. Thus, younger adults typically aim to explore the world, to have new experiences, and to seek out interactions with novel social partners. In contrast, older adults are more likely to appreciate life's fragility and perceive time as "running out" or being limited. This in turn causes them to prioritize present-oriented goals, such as maximizing their emotional well-being. Thus, with age, people focus more on savoring life and deepening existing relationships (Carstensen, 1993, 1995, 2006).

These time-horizon-induced differences in social goals are in turn hypothesized to lead to the positivity effect in attention and memory. When people perceive their time horizons as limited they aim to optimize their emotional well-being. One way that this can be accomplished is by preferentially attending to and remembering positive over negative information (and hence display a positivity effect; see Kryla-Lighthall \& Mather, 2009; Reed \& Carstensen, 2012).

Research has consistently supported socioemotional selectivity theory's prediction that fluctuations in time horizons affect social goals. For example, although there are usually age differences in perceived time horizons, this is not always the case. Some situations, such as the 9/11 attacks on the USA or the SARS (severe acute respiratory syndrome) epidemic in Hong Kong, remind people of all ages that life is fragile. In response to these situations, both younger and older adults prioritized the goal of maximizing their emotional well-being (Fung \& Carstensen, 2006). Similar results emerge in studies that experimentally manipulate time horizons and examine how this affects social preferences. For example, in a study by Fung, Carstensen, and Lutz (1999), some younger adults were asked to reflect on "running out of time" within their current social circle by imagining a hypothetical geographic move. In response to this, these younger adults became indistinguishable from older adults in their social goals; both groups equally prioritized spending time with familiar social partners, an activity that would likely maximize their emotional well-being.
Conversely, older adults who reflected on the possibility of an expanded time horizon (by imagining a medical breakthrough that added 20 years to their life), became indistinguishable from younger adults in their social goals; both groups prioritized spending time with novel social partners, an activity that would likely maximize knowledge-acquisition. Thus, when time horizons are perceived as limited, people focus on maximizing emotional satisfaction regardless of their age. Conversely, when time horizons are perceived as expansive, people focus on knowledge acquisition (see also Carstensen \& Fredrickson, 1998; Fredrickson, 1995; Fung \& Carstensen, 2003).

Although there is clear evidence that shifting time horizons affect social goals, there is less experimental evidence that shifting time horizons also lead to the positivity effect. For instance, one study found that first-year college students looked longer at sad faces than did college seniors. This was interpreted as occurring because the seniors perceived their college time as "running out' due to their upcoming graduations (Pruzan \& Isaacowitz, 2006). Although these results are consistent with socioemotional selectivity theory, this experiment was quasiexperimental in nature and did not directly manipulate time horizons. Without random assignment to conditions, it is not clear whether reductions in time horizons were responsible for the increased positivity. A different problem arose in a study examining the relationship between individual differences in time horizons and visual attention biases on the dot-probe task (Demeyer \& De Raedt, 2013). In this study, no relationship was found between these factors. However, this study also failed to observe an age-related positivity effect, likely because dot-probe tasks provide less power to detect positivity effects than eye tracking (Isaacowitz et al., 2006a). Furthermore, in this study the measure used to assess time horizons had relatively low reliability in the older adult sample. Taken together, these two factors likely reduced the ability of this study to observe a relationship between time horizons and the positivity effect.

Other studies have attempted to examine the impact of time horizons on positivity by experimentally shifting time horizons. However, it is unclear how effective their manipulations of time horizons have been. For example, one study found that students who reflected on their own mortality showed greater accessibility of positive emotional information than control groups (DeWall \& Baumeister, 2007). Likewise, students completing a taste test enjoyed eating a chocolate more when they knew it was the last item they would taste (O'Brien \& Ellsworth, 2012). However, it is not clear whether these manipulations affected positivity by changing time horizons. Similarly, another study attempted to manipulate time horizons by having participants think about events happening in the next 2 days versus in the next 10 years (Demeyer \& De Raedt, 2014). Here, no effect was found on attentional biases. However, it is not clear whether asking participants to shift their focus to the near future versus far future affected perceived time horizons. 
The study with perhaps the cleanest experimental manipulation of time horizons to date (Kellough \& Knight, 2012) provides some evidence consistent with socioemotional selectivity theory. In this study, half of the younger adults imagined that it was their graduation day (i.e., a limited time horizon) and half of the younger adults were given no special instructions. Likewise, half of the older adults imagined a medical breakthrough that provided 20 additional years of life (i.e., an expansive time horizon) and half of the older adults were given no special instructions. All participants then saw emotional faces and indicated which emotion(s) they displayed. Consistent with socioemotional selectivity theory, older adults in the expansive time horizon condition judged ambiguous emotional faces less positively than did older adults in the control condition. However, contrary to expectations, there were no analogous effects for the younger adults. This inconsistency may have been due to differences in the way time horizons were manipulated for the two age groups. For older adults, time horizons were manipulated to be expansive by imagining a lengthened life expectancy. For younger adults, time horizons were manipulated to be limited by imagining their graduation. Although this is the end of a developmental stage, it is also the beginning of new opportunities (as students may now begin careers and families). In addition, the dependent variable in Kellough and Knight (2012) was categorization of facial emotions, a task on which age-related positivity effects are often not found (in part because there are age differences in eye gaze patterns that influence how facial emotions are interpreted; for a review, see Mather, 2016). Use of this categorization task rather than an attentional or memory task may have reduced the likelihood of finding effects.

Thus, although the vast majority of positivity effect studies have been interpreted through the lens of socioemotional selectivity theory, it remains unclear whether or not positivity effects are actually caused by the changes in social goals that arise due to fluctuating time horizons. In addition, to our knowledge, no previous study has directly examined the link between time horizons and the positivity effect within the domain of memory. The goal of the current research was to examine the impact of time horizons on the positivity effect, using the most frequently assessed measure of the effect, namely recall of emotional pictures. In two experiments, we asked participants to complete a writing activity, which directed them to think of time as being either limited or expansive. In Experiment 1, we included both limited and expansive time horizon conditions for both younger and older adults. Experiment 2 also included a control condition, which did not orient participants to think about time in a particular manner. Based upon socioemotional selectivity theory we predicted that regardless of chronological age, thinking about a limited future would lead to enhanced positivity in recall.

\section{Experiment 1}

In Experiment 1 we manipulated the salience of time horizons in both younger and older adult participants via a writing activity. Participants either reflected on how a life expectancy of six remaining months (i.e., a limited time horizon) or 120 years (i.e., an expansive time horizon) would affect their activities and goals. Participants then saw positive, negative, and neutral pictures, which they subsequently tried to recall. Based upon socioemotional selectivity theory, we predicted that our time horizon manipulation should affect the positivity of participants' recall, with enhanced positivity for participants in the limited, compared to expansive, time horizon condition.

\section{Method}

\section{Participants}

A total of 161 individuals ( 81 older and 80 younger adults) from the Los Angeles area participated in this study. Due to a computer failure, data from two older adult participants were lost. We also excluded data from four non-native English speakers (two older and two younger adults), and from two individuals (one older and one younger adult) who did not complete the writing activity (described below) within the allotted 10 minutes (i.e., they were still writing their responses and/or had not answered a question). This left a final sample of 76 older adults and 77 younger adults in the following analyses. Of this final sample, older adults were on average 69.47 years old $(S D=5.27$; range $=61-80$ years $)$ whereas younger adults were on average 20.30 years old $(S D=2.56$; range $=18-34$ years $)$. Older adults had completed more years of education $(M=17.88)$ than younger adults $(M=13.87)$, who were still students at the time of this study, $t(150)=$ $14.21, p<.001, d=2.32{ }^{1}$

There were significantly more men in the older adult sample $(50 \%)$ than in the younger adult sample $(18 \%)$. However, randomization to the experimental writing condition was done separately for men and women in each

\footnotetext{
${ }^{1}$ For the three older adult participants who indicated they had " $16+$ years" of education we assumed 17 years of education. For the one older adult participant who indicated they had completed trade school we assumed 14 years of education. One older adult participant did not answer this question.
} 
age group. Because of this, within each age group there was an approximately equal number of men assigned to the limited time horizon condition (18 older and seven younger men) as to the expansive time horizon condition (20 older and seven younger men). None of the reported patterns of results changed when including gender as a covariate.

Participants were recruited through the University of Southern California (USC) psychology participant pool and a list of volunteers associated with the USC Leonard Davis School of Gerontology. Upon completion of the study, participants were compensated either $1 \mathrm{credit} / \mathrm{h}$ towards their psychology course requirements or US $\$ 15 / \mathrm{h}$.

\section{Procedure}

Participants first completed a demographics form. They were then randomly assigned to either the limited time horizon condition or to the expansive time horizon condition. The manipulations used in these conditions were modeled after previous work by Fung and colleagues (1999) examining how time horizons affect social goals.

In the Limited Time Horizon condition participants completed a writing activity in which they reflected on a shortened life expectancy and the need to spend more time focusing on the present (for the full text, see Appendix 1). These participants spent at least 5 minutes writing about how knowing they only had 6 months left to live would change their current spending and saving plans as well as the activities they spent time on. They also described how they would like to spend the last day of their life, and what their life goals would be if they knew they only had 6 more months left to live.

In contrast, in the Expansive Time Horizon condition participants completed a writing activity in which they reflected on an increased life expectancy (for the full text, see Appendix 1). These participants spent at least 5 minutes writing about how knowing they would live in good health to the age of 120 years would change their spending and saving plans as well as the activities they spent time on. They also described how they would like to spend their days after they reached the age of 100 years, and what their life goals would be if they knew they would live to be 120 years old.

Participants in both conditions were left alone for 5 minutes to complete the writing activity. If participants were not finished when the experimenter returned, they were given up to 5 additional minutes to finish answering the questions. For example transcriptions from both younger and older adults in each of the conditions, see Appendix 1. For analyses of the linguistic content of participants' writing activity responses, please see the Supplementary Materials.

To determine the efficacy of this writing activity in changing time horizons we conducted a supplementary study. Here, we recruited an additional set of 131 younger adults $(M=$
25.17 years old; range $=18-35)$ and 120 older adults $(M=$ 57.72 years old; range $=50-74)$ from Amazon's Mechanical Turk website. ${ }^{2}$ Participants were randomly assigned to complete either the limited or expansive time horizon writing activity that was just described. They then used a sliding scale to indicate how far they felt they had progressed in their life. Results of a 2 (Age group) $\times 2$ (Time horizon) ANOVA showed a significant main effect of age, $F(1,247)=457.81, M S E=190.47, p<.001$, $\eta_{p}{ }^{2}=.65$. Not surprisingly, older adults felt they had less time remaining in life than did younger adults. Of relevance to this study, there was also an effect of time horizon condition, $F(1$, $247)=3.74, M S E=190.47, p=.05, \eta_{p}{ }^{2}=.02$, which did not interact with age, $F(1,247)=2.29, M S E=190.47, p=.13, \eta_{p}{ }^{2}=$ .01. Participants in the limited time horizon condition felt they had less time remaining in life than did participants in the expansive time horizon condition. Thus, results from this supplementary study supports that our manipulation shifts time horizons similarly across age groups.

Within Experiment 1, immediately after the writing activity, participants completed an emotional memory task. Participants were shown a series of 70 pictures (drawn from those used by Mather \& Knight, 2005, Experiment 2, and selected to be distinguishable from one another based upon verbal descriptions). Of these, 14 pictures were neutral, 28 were positive, and 28 were negative in valence. The majority of the pictures came from the International Affective Picture System (IAPS; Lang, Bradley, \& Cuthbert, 1999). ${ }^{3}$ However, two neutral pictures came from additional sources. Within the positive and negative valence categories, an equal number (14) of pictures were low and high in emotional arousal. Previous results from Mather and Knight (2005, Experiment 2) showed that younger and older adults did not differ in their valence or arousal ratings of these pictures. Pictures appeared in a single random order at a rate of one picture every $2 \mathrm{~s}$. As in Mather and Knight (2005), participants were asked to view the pictures the same way that they would watch television and were also informed that their memory for the pictures would later be tested. After viewing the pictures, participants completed a self-paced free recall test. Here, participants described aloud as many of the pictures as they could recall

\footnotetext{
$\overline{{ }^{2} \text { An additional }} 39$ participants completed this supplementary study but were excluded either because their IP address was associated with multiple responses, they were not a native English speaker, or their speed of completing the study suggested they did not answer the questions seriously.

${ }^{3}$ Negative high arousal pictures: IAPS numbers 3110, 3180, 3350, 3500, $6230,6350,9040,9410,9500,9611,9621,9810$, and 9911. Negative low arousal pictures: IAPS numbers $1112,1275,2490,2590,2700,2750$, 3300, 5970, 6000, 9001, 9007, 9330, 9470, and 9830. Neutral pictures: IAPS numbers 2200, 2840, 5510, 5731, 5920, 7010, 7090, 7130, 7150, $7170,7217,7640$, and two additional pictures from additional sources. Positive high arousal pictures: IAPS numbers 4220, 4608, 5260, 5460, 5470, 5621, 5629, 7270, 7502, 8030, 8300, 8370, 8470, and 8501 . Positive low arousal pictures: IAPS numbers 1463, 1590, 2091, 2260, 2352, 2540, 2550, 2650, 4700, 5220, 5300, 5660, 5982, and 7480 .
} 
while the experimenter sat beside them and typed their responses into the computer. The transcribed responses were visible to both the experimenter and the participants throughout the memory test. There were no audio and video recordings.

\section{Results}

A picture was scored as correctly recalled if the participant provided a description that a rater determined to clearly match the picture. The recalled pictures were then classified by valence based upon their IAPS ratings. In total, 78 of the 3,223 responses $(2.4 \%)$ could not be matched to presented pictures. Most of the unscored responses were descriptions that were too general to be matched to a picture (e.g., "someone laughing or smiling," "scenery picture"). We next conducted a 2 (Age group) $\times 2$ (Time horizon) ANOVA on the number of responses provided by each participant that could not be scored. Results of this analysis showed that older adults were more likely to provide responses that could not be scored ( $M=$ 0.67 responses per participant) than younger adults $(M=.32)$, $F(1,149)=6.20, M S E=0.74, p=.01, \eta_{p}{ }^{2}=.04$. However, there was no main effect of time horizon condition, $F<1$, or interaction between time horizon condition and age, $F<1$ on the number of responses that could not be scored. To ensure that the ratings were reliable, a second rater independently coded for the presence or absence of each picture in all of the recall protocols. Reliability with the primary rater was extremely high, Cohen's kappa $=0.96, p<.001$.

We next examined how age and time horizon condition affected the total number of pictures recalled (collapsing across valence; see Table 1). As expected, a 2 (Age group) $\times$ 2 (Time horizon) ANOVA on total recall showed no significant main effect of time horizon condition, $F(1,149)=0.02$, $M S E=62.26, p=.88, \eta_{p}{ }^{2}<.001$, and no interaction between age group and time horizon condition, $F(1,149)=0.97, M S E$ $=62.26, p=.33, \eta_{p}{ }^{2}=.006$. However, surprisingly within this analysis there was no main effect of age group, $F(1,149)=$ $0.39, M S E=62.26, p=.54, \eta_{p}^{2}=.003$. Further analyses showed that this null effect was likely due to age differences in time spent on the self-paced free recall test. Within a 2 (Age group) $\times 2$ (Time Horizon condition) ANOVA on recall times there was a main effect of age, $F(1,149)=23.34, M S E=$ 16207.57, $p<.001, \eta_{p}{ }^{2}=.14$, such that older adults elected to spend more time on the test $(M=313.70 \mathrm{~s})$ than did younger adults $(M=214.22 \mathrm{~s})$. Recall time was in turn positively correlated with the amount recalled, $r=.59, p<.001$. Importantly, after accounting for recall time, a standard age difference in total recall emerged. Within a 2 (Age group) $\times 2$ (Time horizon) ANCOVA on total recall there was a significant main effect of age such that older adults recalled significantly fewer pictures (adjusted $M=18.11$ ) than younger adults (adjusted $M=21.07), F(1,150)=7.54, M S E=$ $38.73, p=.007, \eta_{p}{ }^{2}=.05$. However, within this ANCOVA there was still no main effect of time horizon condition, $F(1$, $148)=1.10, M S E=38.92, p=.30, \eta_{p}{ }^{2}=01$, and no interaction between age group and time horizon condition, $F(1,148)=$ $0.15, M S E=38.92, p=.70, \eta_{p}{ }^{2}=.001$, suggesting that our manipulation did not affect overall levels of recall.

The positivity effect has been defined as an age-by-valence interaction, in which older adults' recall is proportionally more positive, and less negative, than younger adults' recall (Kryla-Lighthall \& Mather, 2009). To capture this shift towards positive and away from negative, in the current study we calculated the relative positivity of each participant's recall. We defined this as the number of positive pictures recalled minus the number of negative pictures recalled divided by the total number of pictures recalled. Positive scores indicate a propensity to recall the positive rather than the negative pictures. In contrast, negative scores indicate a propensity to recall the negative rather than positive pictures. A key strength of this relative index of positivity is that it also accounts for the large variability in total recall, which in the current study ranged from four to 49 pictures. For the total number of items recalled from each valence category as a function of age and time horizon condition, see Table 1.

We next turned to the primary purpose of Experiment 1: Did the manipulation of time horizons affect whether people preferentially recalled positive or negative pictures? To answer this, we conducted a 2 (Age group) $\times 2$ (Time horizon)

Table 1 Total number of pictures recalled as a function of participant age, time horizon condition, and picture valence in Experiment 1

\begin{tabular}{lllll}
\hline & Younger adults & & Older adults \\
\cline { 2 - 5 } & $\begin{array}{l}\text { Expansive time } \\
\text { horizon }\end{array}$ & $\begin{array}{l}\text { Limited time } \\
\text { horizon }\end{array}$ & $\begin{array}{l}\text { Expansive time } \\
\text { horizon }\end{array}$ & $\begin{array}{l}\text { Limited time } \\
\text { horizon }\end{array}$ \\
\hline Positive pictures & $8.49(3.96)$ & $8.18(3.79)$ & $8.82(3.53)$ & $9.84(3.34)$ \\
Negative pictures & $9.15(3.79)$ & $8.13(4.05)$ & $8.10(3.31)$ & $2.36(1.63)$ \\
Neutral pictures & $2.10(1.50)$ & $2.37(2.07)$ & $19.22)$ \\
Total recall & $19.74(7.87)$ & $18.68(8.41)$ & $19.28(7.18)$ & $20.73(8.08)$ \\
\hline
\end{tabular}

Note: There were 28 positive, 28 negative, and 14 neutral pictures, for a total of 70 pictures 
ANOVA on the positivity of recall. Consistent with our hypotheses, within this analysis there was a significant main effect of time horizon condition, $F(1,149)=5.17, M S E=$ $.04, p=.024, \eta_{p}{ }^{2}=.03$, which did not interact with age, $F<$ 1. As can be seen in Fig. 1, the positivity of participants' recall was higher in the limited time horizon condition than in the expansive time horizon condition.

Although the effect of our time horizon manipulation was age-invariant, within this ANOVA there was also a main effect of age, indicative of an age-related positivity effect, $F(1,149)=$ 10.04, MSE $=.04, p=.002, \eta_{p}^{2}=.06$. As shown in Fig. 1, within each of the time horizon conditions the positivity of older adults' recall was higher than that of younger adults' recall.

An interesting additional observation is that the magnitude of the positivity bias was dependent upon participants' overall levels of recall. Lower levels of recall were associated with significantly higher levels of positivity in the limited time horizon condition (partial correlation controlling for age group: $r=$ $-0.26, p=0.02$ ) and marginally with lower levels of positivity in the expansive time horizon condition (partial correlation controlling for age group: $r=.20, p=.09$ ). Thus, the biases prompted by the time horizon manipulation were especially apparent in the participants who performed poorly on the memory test.

\section{Discussion}

In Experiment 1 younger and older adults reflected on either a limited or expansive time horizon before completing an emotional memory task. Results were consistent with socioemotional selectivity theory. Regardless of chronological age, reflecting on a limited time horizon was associated with higher positivity in recall compared to reflecting on an expansive time horizon. Although the results of Experiment 1 were

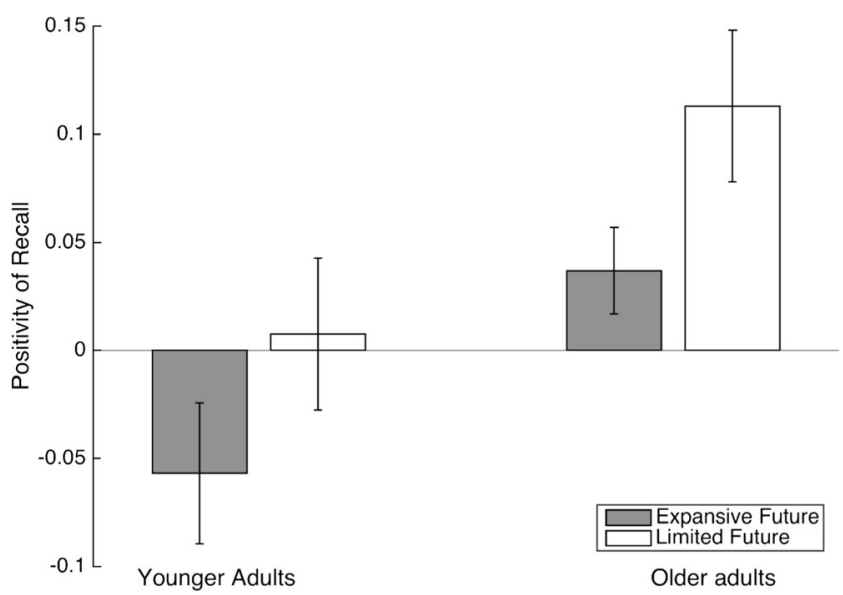

Fig. 1 Positivity of participants' recall as a function of age and time horizon condition in Experiment 1. Positivity was calculated as the number of positive pictures recalled minus the number of negative pictures recalled divided by the total number of pictures recalled. Error bars represent \pm SE mean consistent with socioemotional selectivity theory's hypotheses, there were also some limitations to this study. First, we did not include a control condition. Thus, it is unclear whether our limited time horizon manipulation increased the positivity of recall, whether the expansive time horizon manipulation decreased the positivity of recall, or whether both effects simultaneously occurred. Second, we did not include measures of mood. It is possible that reflecting on the possibility of having only 6 months to live induces a negative mood state, which in turn might affect the positivity of participants' recall. It is also possible that reflecting on the possibility of living up to 120 changes individuals' affective states; however, the direction of this effect was less clear. In Experiment 1 there was a variety of responses to the expansive time horizon manipulation. Some participants wrote about using this extended lifespan to pursue pleasurable novel activities (e.g., travelling the world, becoming a teacher). However, other participants wrote about plans to be frugal (in order to financially support this extended lifespan) and about fears that their loved ones would not have a similarly long lifespan.

\section{Experiment 2}

To address the two limitations of Experiment 1 outlined above, we conducted a second experiment. Here, we included a control writing condition, in which participants were not oriented to think about time in a particular manner, but rather described activities that they had completed that day. This allowed us to determine whether participants in the limited time horizon condition show enhanced positivity in recall, whether participants in the expansive time horizon condition show reduced positivity in recall, or whether both effects occur simultaneously. We also included assessments of mood. This allowed us to determine whether reflecting on a limited future induces a negative mood state, and how this in turn affects the positivity of participants' recall.

\section{Method}

\section{Participants}

A total of 150 adults (46 women) were recruited via Amazon's Mechanical Turk (mTurk), an online portal that connects people willing to do short web-based tasks with people who need those tasks completed. Data obtained from mTurk participants have high test-retest reliability (Buhrmester, Kwang, \& Gosling, 2011) and do not significantly differ from data obtained from in-person laboratory study participants (Paolacci, Chandler, \& Ipeirotis, 2010) Participants were compensated US\$1 upon completion of the study.

Of the 150 participants, we excluded 13 who reported having participated in a prior mTurk experiment using the same 
emotional pictures and 24 who reported a computer error in which the file names of the emotional pictures appeared alongside the pictures. ${ }^{4}$ We also excluded two participants who failed to recall any of the critical pictures. This left a final sample of 111 participants: 39 in the expansive time horizon condition, 33 in the limited time horizon condition, and 39 in the control condition. This sample was on average 31.88 years old $(S D=9.52)$. Although participants ranged in age from 20 to 71 years, we did not recruit participants based upon their age. Because of this, the distribution of age was not normally distributed: $47.7 \%$ of participants were aged 20-29, $33.4 \%$ were aged 30-39, $13.5 \%$ were aged 40-49, $4.5 \%$ were aged $50-59$, and only $0.9 \%$ were aged 60 years or older. Participant age did not differ between the three experimental conditions, $F(1,108)=0.78, M S E=91.48, p=.46, \eta_{p}{ }^{2}=.01$.

By chance, the three experimental conditions marginally differed in the number of women assigned to them, $F(2,108)=$ $2.94, M S E=0.24, p=.06, \eta_{p}{ }^{2}=.05$. There was a numerically lower proportion of women in the limited time horizon condition $(24.2 \%)$ than in either the expansive time horizon $(48.7 \%)$ or control condition (48.7\%). However, Bonferonni-corrected post-hoc comparisons showed that these differences were not statistically significant. The reported patterns of results do not change when including gender as a covariate.

Participants had a broad range of educational backgrounds: $0.9 \%$ had completed some high school, $11.7 \%$ had a high school diploma, $32.4 \%$ had "some college," $17.1 \%$ had a $2-$ year Associates level college degree, $33.3 \%$ had a 4-year Bachelor's level college degree, 3.6 \% had a Master's degree, and $0.9 \%$ had a Ph.D., J.D., or M.D. degree. By chance, education significantly differed among the three experimental conditions, $F(2,108)=4.06, M S E=1.35, p=.02, \eta_{p}{ }^{2}=.07$. Bonferroni-adjusted post-hoc analyses showed that this was because participants randomly assigned to the control condition had significantly higher levels of educational attainment than those assigned to the limited time horizon condition, $p=$ .02. No other pairwise comparison was significant (all $p$ 's $>$ .29). The reported patterns of results do not change when including education as a covariate.

\section{Procedure}

As a baseline measure of mood, participants first completed the Positive and Negative Affective Scale (PANAS; Watson, Clark, $\&$ Tellegen, 1988). This 20-item questionnaire lists ten positive and ten negative emotional adjectives and participants rate the extent to which they are currently feeling each adjective.

\footnotetext{
${ }^{4}$ Pretesting revealed that the file names of pictures sometimes appeared, and this was associated with a delay in the pictures loading. Because of this, at the end of this study we explicitly asked all participants whether or not the file names had appeared during encoding.
}

Participants were then randomly assigned to either the limited time horizon, expansive time horizon, or control condition. These conditions differed only in the writing activity that was next completed. The instructions for the writing activities used in the limited and expansive time horizon conditions were identical to those used in Experiment 1 with one exception - here, the sentences stating that participants should assume good health were printed in bold and underlined. As described in the Supplementary Material, in Experiment 1 participants in the expansive time horizon condition were more likely to write about their health despite the fact that instructions in both conditions stated that participants should assume good health. In Experiment 2, we examined whether this linguistic difference would remain when these statements were highlighted. See the Supplementary Materials for more details on these linguistic analyses.

In contrast to the other two conditions, the control condition's writing activity did not include a preamble asking participants to think about time in a specific manner. Rather, these participants responded to four questions about their current daily activities (see Appendix 2), which were designed to be similar in topic to those answered in the limited and expansive time horizon conditions (see Appendix 1). For example, whereas participants in the limited and expansive time horizon conditions speculated on how their daily activities would be affected by changes in life expectancy, participants in the control condition listed the activities they had completed that day. For analyses of the linguistic content of participants' writing activity responses, see the Supplementary Materials.

Immediately after the writing activity, participants in all three conditions indicated their mood using a sliding scale. Responses could range from 0 (very negative mood) to 100 (very positive mood).

Participants next completed an emotional picture memory task. The picture stimuli used in this task were seven positively-valenced and seven negatively-valenced pictures drawn from the IAPS (Lang et al., 1999). ${ }^{5}$ They were all low in arousal, and arousal level did not differ between the positive and negative pictures. In contrast to Experiment 1, in Experiment 2 we used an incidental encoding task. This procedural change was made for two reasons. First, it ensured that participants did not make notes about the pictures for the upcoming memory test. Second, it was expected to increase the likelihood of observing a positivity effect; results of a recent meta-analysis suggest that the positivity effect is larger during incidental compared to intentional encoding (Reed, et al., 2014). During the incidental encoding task pictures were shown in a single random order. The picture slideshow progressed

\footnotetext{
${ }^{5}$ Negative pictures: IAPS numbers $1112,1275,2490,9001,9415$, and 9830. Positive pictures: IAPS numbers $1463,2260,2540,2550,2650$, 5660 , and 7350 . There were images of people in four of the negative and in four of the positive pictures.
} 
automatically, and participants could not go back and review pictures after they had disappeared. Each picture was shown with either a red or a yellow border, and participants were asked to indicate the border's color. Since this was an online study, this ensured that participants attended to all of the pictures during the encoding period. Each picture was shown for $5 \mathrm{~s}$. This was an increase in exposure time compared to Experiment 1 (in which each picture was shown for only $2 \mathrm{~s}$ ). This procedural change ensured that participants had adequate time to both note the color of the border and also attend to the content of each picture. Across participants each picture appeared equally often with a red border as it did with a yellow border. To buffer against primacy and recency effects, we also included four non-critical neutral pictures, two of which appeared at the beginning of the slideshow and two at the end. Immediately after viewing the pictures, participants completed a surprise, self-paced, free recall test. Here, they typed short descriptions of as many of the pictures as they could recall.

Finally, at the end of the study participants provided demographics information and also indicated whether they had encountered any technical problems or had seen the emotional picture stimuli in a previous experiment.

\section{Results}

A picture was scored as correctly recalled if the participant provided a description that matched the picture. Recalled pictures were classified by valence according to their IAPS ratings. Only 33 of the 690 responses could not be scored (4.8\%); a single-factor betweengroups ANOVA on the number of non-scored responses provided by each participants showed no significant difference among the three conditions, $F(1,108)=1.31$, $M S E=0.37, p=.28, \eta_{p}{ }^{2}=.02$. To ensure reliability of the ratings, a second rater independently coded for the presence of the 14 critical pictures in each of the recall protocols. As in Experiment 1, reliability with the primary rater was high, Cohen's kappa $=0.93, p<.001$. A single-factor between-groups ANOVA confirmed that there was no significant difference among the three conditions in the total number of pictures recalled, $F$ $(2,108)=0.45, M S E=5.77, p=.64, \eta_{p}{ }^{2}=.008$ (see Table 2).

We next turned to the first primary aim of Experiment 2: Did the positivity of recall differ between the three conditions? To answer this, we conducted a single-factor between-groups ANOVA on the relative positivity of participants' recall. As in Experiment 1, we defined this as the number of items recalled that were positive minus the number that were negative divided by the total number of items recalled (for the number of items recalled as a function of valence and time horizon condition see Table 2). As shown in Fig. 2, the positivity of participants' recall significantly differed among these three conditions, $F(2,108)=3.17, M S E=.20, p=.046, \eta_{p}{ }^{2}=.06$. In follow-up independent t-tests, participants' recall in the limited time horizon condition was marginally more positive than that of participants in the expansive time horizon condition, $t(70)=1.93, p=.057, d=.46$, and significantly more positive than that of participants in the control condition, $t(70)=2.50$, $p=.015, d=.60$. In contrast, the positivity of recall did not differ between participants in the expansive time horizon and control conditions, $t(76)=0.48, p=.63, d=.11$. Thus, whereas a focus on limited time horizons increased the positivity of recall, a focus on expansive time horizons did not decrease it.

As in Experiment 1, the magnitude of the positivity bias in the limited time horizon condition was negatively associated with the participants' overall levels of recall $(r=-0.69, p<.001)$, such that lower overall recall was related to a stronger positive bias. However, unlike in Experiment 1, there was no significant correlation between total recall levels and the magnitude of the positivity effect in the expansive future condition $(r=-0.21$, $\mathrm{p}=.20$ ). There was also no relationship between these variables in the control conditions $(r=0.07, \mathrm{p}=.65)$.

\section{Mood and free recall}

Our second aim was to test the role of mood in modulating our results. We hypothesized that reflecting on a limited future would induce a negative mood. However, it was unclear how this would in turn affect the positivity of recall. On the

Table 2 Total number of pictures recalled as a function of time horizon condition and picture valence in Experiment 2

\begin{tabular}{llll}
\hline & Expansive time horizon condition & Control condition & Limited time horizon condition \\
\hline Positive pictures & $2.33(1.28)$ & $2.56(1.47)$ & $2.55(0.94)$ \\
Negative pictures & $2.33(1.63)$ & $2.49(1.52)$ & $2.00(1.56)$ \\
Total recall & $4.67(2.35)$ & $5.05(2.60)$ & $4.55(2.21)$ \\
\hline
\end{tabular}

Note: There were seven positive and seven negative pictures, for a total of 14 pictures. Numbers in parentheses represent standard deviations. Of note, recall levels were lower in Experiment 2 than in Experiment 1 because of procedural differences at encoding. Whereas encoding was incidental during Experiment 2, it was intentional during Experiment 1. This change to incidental encoding likely also led to the larger positivity effects in Experiment 2 than in Experiment 1 (see Reed et al., 2014) 


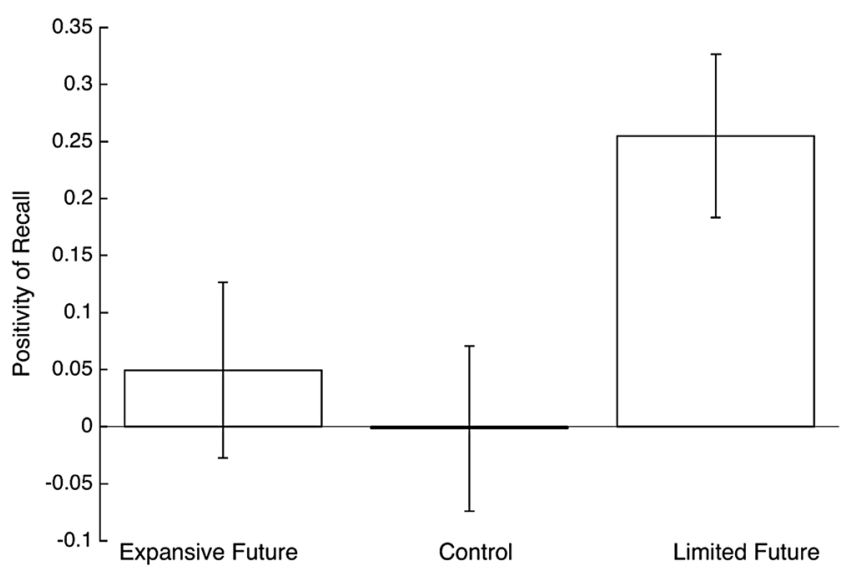

Fig. 2 Positivity of participants' recall as a function of time horizon condition in Experiment 2. Positivity was calculated as the number of positive pictures recalled minus the number of negative pictures recalled divided by the total number of pictures recalled. Error bars represent $\pm \mathrm{SE}$ mean

one hand, there could be a negative correlation between these factors; a reduced mood in the limited time horizon condition could lead to enhanced positivity if people selectively recall the positive pictures as a means of enhancing positive emotion. In other words, mood could serve as a mediating variable and explain why our time horizon manipulation affected the positivity of participants' recall. On the other hand, there could be a positive correlation between these factors; a reduced mood could lead people to engage in mood-congruent processing and attenuate the positivity of their recall. In other words, a reduced mood in the limited time horizon condition could serve as a suppressor variable, reducing the predictive strength of our time horizon manipulation in accounting for the subsequent positivity of participants' recall.

To address these questions, we first examined whether our time horizon manipulation affected mood. At the outset of the study, mood (as assessed via the PANAS) did not differ between the conditions; a single-factor between-group ANOVA revealed no significant differences in either positive or negative PANAS scores among the three conditions, $F(1,108)=0.71, M S E=$ $74.35, p=.50, \eta_{p}{ }^{2}=.01$ and $F(1,108)=0.35, M S E=16.45, p=$ $.70, \eta_{p}{ }^{2}=.01$, respectively. In contrast, after completing the writing activity, mood (as assessed via a sliding scale from 0 to 100$)$ significantly differed among the conditions, $F(2,108)=$ 4.60, MSE $=501.17, p=.01, \eta_{p}{ }^{2}=.08 .{ }^{6}$ Follow-up independent t-tests showed that after the writing activity, participants in the limited time horizon condition were in a significantly worse $\operatorname{mood}(M=60.18)$ than participants in either the expansive time horizon $(M=72.41), t(70)=-2.08, p=.04, d=.50$, or control condition $(M=75.56), t(70)=-3.28, p=.002, d=.79$. Mood

\footnotetext{
${ }^{6}$ One limitation of this study is that the mood measures used pre- and post-writing differed. Although participants in the three conditions did not significantly differ in their positive or negative PANAS scores prior to the writing activity, it is possible that they would have differed in their responses to the one-question sliding scale measure of mood.
}

did not vary between participants in the expansive time horizon and control conditions, $t(76)=-.63, p=.53, d=.14$. Thus, whereas a focus on limited time horizons induced a negative mood, a focus on expansive time horizons did not significantly affect mood either positively or negatively.

How did mood affect the positivity of participants' recall? Across the three conditions there was a positive correlation between these two variables; the higher the participants' mood the greater their positivity of recall, $r=.23, p=.015$. Thus, collapsing across the three conditions, people engaged in mood-congruent processing and had a tendency to recall the positive pictures when in a positive mood.

In summary, the previous results have shown (a) that participants in the limited time horizon condition were in a worse mood, (b) that a lower mood was generally associated with lower positivity in recall, (c) that despite their lower mood (and thus their propensity to engage in mood-congruent processing and display a negativity bias), participants in the limited time horizon condition surprisingly had higher positivity in recall than participants in the other two conditions. Taken together, these results suggest that changes in mood do not mediate the relationship between our time horizon manipulation and the positivity of recall. Rather, reflecting on a limited future appears to enhance the positivity of recall (presumably because of reduced time horizons) but the strength of this relationship may be suppressed by the accompanying negative mood that is induced. To test this, we next used the logic of a mediation analysis to examine whether the strength of the direct path between our time horizon manipulation and the positivity of participants' recall would change after accounting for mood (see Fig. 3). In a typical mediation analysis, the strength of the direct path is weakened after accounting for the intervening (mediating) variable. In contrast to this, we expected the strength of the direct path to be enhanced after accounting for the intervening (suppressive) variable.

As shown in Fig. 3, in separate regression analyses we found that time horizon condition (entered as 0 for either the expansive time horizon or control condition and as 1 for the limited time horizon condition) was significantly related to both mood and the positivity of recall. We then conducted another regression analysis where time horizon condition and mood were simultaneously entered as predictors of the

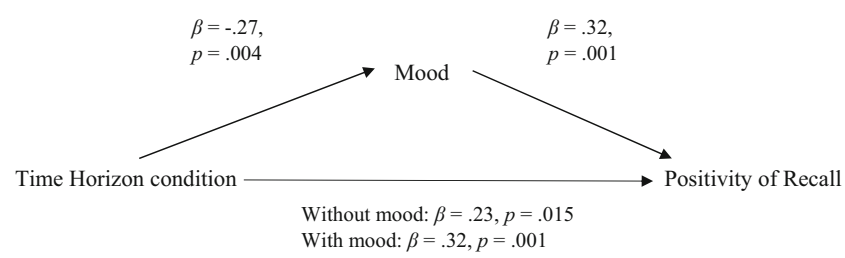

Fig. 3 The relationship between time horizon condition, mood, and the positivity of recall in Experiment 2. When accounting for mood, the relationship between time horizon condition and the positivity of recall was significantly strengthened 
positivity of recall. Here, both time horizon condition and mood predicted the positivity of recall. Furthermore, the relationship between time horizon condition and the positivity of recall was significantly strengthened after accounting for mood, Sobel test $\mathrm{Z}=-2.20, p=.028$.

\section{Discussion}

Replicating Experiment 1 results, participants in the limited time horizon condition displayed higher positivity than participants in the expansive time horizon condition. Novel to Experiment 2, we also included a control writing condition. Compared to this condition, we found that thinking about a limited future increased the positivity of recall. In contrast, a focus on expansive time horizons did not decrease it.

We also examined the role of mood in modulating the observed effects. We found that reflecting on a limited future lowered mood. However, this did not explain why participants in the limited time horizon condition had enhanced positivity in recall. Overall, our results showed that reflecting on a limited life expectancy enhanced the positivity of recall, likely by reducing time horizons and increasing participants' motivation to optimize their emotional experience. However, this enhanced positivity was attenuated by the accompanying negative mood state that was induced by reflecting on having only 6 months left to live.

\section{General discussion}

Across a variety of cognitive domains, older adults show a relative preference (compared with younger adults) towards positive and/or away from negative information. This is known as the positivity effect (Charles et al., 2003), and is typically explained by socioemotional selectivity theory (e.g., Carstensen, Isaacowitz, \& Charles, 1999). According to this view, as people get older they begin to perceive time as more limited and this causes them to prioritize goals related to enhancing their current emotional satisfaction. One way this can be achieved is by focusing on and remembering positive (rather than negative) information (i.e., by displaying a positivity effect; see Kryla-Lighthall \& Mather, 2009; Reed \& Carstensen, 2012).

Although age-related changes in time horizons are frequently theorized to cause the positivity effect, previous research either did not directly test this assumption or yielded unclear results (Demeyer \& De Raedt, 2013, 2014; DeWall \& Baumeister, 2007; Pruzan \& Isaacowitz, 2006, but see Kellough \& Knight, 2012). In two experiments we examined the effect of manipulating time horizons on emotional picture memory recall. Results of both experiments were consistent with socioemotional selectivity theory. In Experiment 1 we found that reflecting on a limited time horizon was associated with enhanced positivity in recall compared to reflecting on an expansive time horizon. This was equally true for younger and older adults. In Experiment 2 we replicated the effect of time horizon on memory positivity. Novel to this study, we also included a control condition. Compared to this control condition, reflecting on a limited time horizon increased the positivity of recall. In contrast, reflecting on an expansive time horizon did not decrease it.

In Experiment 2 we also demonstrated that the effects of our time horizon manipulation on the positivity in recall could not be explained by changes in mood. Not surprisingly, reflecting on a limited future reduced mood. However, despite this mood effect, limiting time horizon increased memory positivity. Accounting for the variance due to mood significantly increased the predictive power of our time horizon manipulation in explaining the magnitude of the positivity of recall. These results suggest that while the limited time horizon manipulation both decreased mood and increased memory positivity, it is unlikely that the increased positivity effect in the limited time horizon condition is due to negative mood in younger adults. In addition, mood also cannot explain the results from Experiment 1. Previous research has shown that whereas younger adults display mood-congruent effects, older adults display moodincongruent effects (Isaacowitz, Toner, Goren, \& Wilson, 2008). Thus, if our limited time horizon writing manipulation also induced a negative mood state in Experiment 1, it should lead to increased negativity in recall in younger adults (a mood-congruent effect) but to increased positivity in recall in older adults (a mood-incongruent effect). However, this was not the pattern of results that was observed in Experiment 1. Rather, the effects of our writing activity in Experiment 1 were age-invariant. Taken together, our results suggest the role of perceived time horizon rather than mood in the positivity effect.

There are limitations to our studies that should be addressed in future research. First, as in previous studies that have examined the relationship between time horizons and the positivity effect in attention (e.g., DeWall \& Baumeister, 2007; Kellough \& Knight, 2012; Pruzan \& Isaacowitz, 2006), in the current study we chose not to directly measure whether time horizons differed between our conditions. We reasoned that directly assessing time horizons could cause participants to notice the effect of the writing activity on their perceptions of time. This could then cause them to mentally "reset" their time horizons to their pre-experiment levels, thereby leaving them unaffected by the manipulation during the subsequent memory task. In Experiment 2, assessing time horizons would also have caused participants in the control condition to begin thinking about time differently. Although our supplementary study (see Experiment 1, Method) showed that our writing activity was effective in changing time horizons, future research should examine the role of perceived time horizons in the positivity of recall directly. 
Second, participants in Experiment 2 were almost all younger adults. For these participants, thinking about a limited future enhanced the positivity of recall but thinking about an expansive future did not reduce the positivity of recall (relative to the control condition). However, it is possible that a different pattern would emerge for older adult participants. Whereas shortening younger adults' time horizons may be easier than lengthening them, the reverse may be true for older adults. Because of this, the positivity of older adults' recall may be equivalent in the limited time horizon and control condition, but less positive in the expansive time horizon condition.

Third, although we included measures of mood in Experiment 2, we did not assess mood after the recall test. According to socioemotional selectivity theory, positivity effects emerge as a means of regulating emotions. Because of this, individuals who displayed enhanced positivity in their recall should have also shown the greatest stability or improvement in their mood over the course of the experiment (e.g., Isaacowitz, Toner, \& Neupert, 2009; Kennedy et al., 2004). Although the focus of these studies was to demonstrate a link between time horizons and the positivity of recall, future research should also examine how time horizons and the positivity effect may together influence participants' affective states.

Of note, although the age-related positivity effect is typically explained according to socioemotional selectivity theory (e.g., Carstensen, et al., 1999), at least two other theoretical frameworks have also been put forward. First, Cacioppo and colleagues' (2011) aging-brain model proposes that there is age-related neural degeneration of the amygdala and this results in dampened amygdala responsiveness to negative stimuli. This in turn decreases the preferential processing advantage that typically occurs for negative stimuli (see Baumeister, Bratlavsky, Finkenauer, \& Vohs, 2001) and leads to agerelated positivity effects. However, the results of our experiments cannot be explained by this framework; the brief time horizon manipulation task used in the current experiments would not cause amygdala neural degeneration. Other evidence also argues against this aging-brain model of older adults' positivity effect (Nashiro, Sakaki, \& Mather, 2012).

Second, according to Labouvie-Vief's $(2003,2009)$ dynamic cognition-emotion integration theory people have two modes of processing affective information. One mode is affect optimization, which refers to the tendency to process the information in a manner that increases positive affect. The second mode is affect complexity, which is the ability to coordinate experiencing both positive and negative affective states. According to dynamic integration theory these two modes are dynamically coordinated such that when one decreases the other tends to increase (Labouvie-Vief \& Medler, 2002). Because of age-related cognitive declines, older adults are proposed to have less affective complexity (see Labouvie-Vief, Diehl, Jain, \& Zhang, 2007). This in turn should lead to increases in affect optimization, and hence an age-related positivity effect.
However, this theory cannot readily explain the current results. It is unclear why reflecting on a limited future would affect cognitive abilities or reduce affective complexity. Thus, our results do not support the dynamic integration theory but rather fit with socioemotional selectivity theory.

In summary, a large number of studies have demonstrated a positivity effect in older adults' attention and memory (Reed et al., 2014). However, to our knowledge, the current experiments are the first to explicitly test socioemotional selectivity theory hypothesis that a direct manipulation of time horizons should influence the positivity of participants' recall. Results from two experiments support socioemotional selectivity theory; reflecting on a limited future enhanced the positivity of both younger and older adults' recall. Thus, the fact that older adults' recall is typically more positive than younger adults' recall may index naturally shifting goals with age.

Acknowledgments Thanks are due to Albert Aboseif, Kaevon Brasfield, Lauran Evans, Sierra Niblett, Henry Tranton, and Amanda Webb for research assistance.

\section{Compliance with ethical standards}

Funding This work was supported by grants from the National Institute on Aging (grant numbers R01-AG025340, R01-AG038043, and R01AG046464) and from the European Commission (FP7-PEOPLE-2013-CIG).

Appendix 1 Full instructions and writing prompt questions, along with example responses from both younger and older adults, in the expansive time horizon and limited time horizon writing conditions of Experiment 1.

Expansive time horizon condition instructions: "People keep living longer and longer, yet official norms for retirement ages have not shifted. There are many more centenarians today than there were 20 years ago, and it is even possible that you might live to be 120 . Yet much research shows that we spend too little time planning for a long future. As you answer the following questions, please plan for a future in which you live to be 120 . Assume you will be in good health.”

\section{Example responses from a younger adult in the expansive time horizon condition:}

1. How would this [knowing you will live to 120] change your spending or saving?

Rather than splurging on luxury items, I would try to be more practical in my spending and to save more to make sure that I am able to live comfortably until age 120 .

2. How would this [knowing you will live to 120] change what activities you spend time on?

I would try to dedicate more time to regularly undergoing physical exercise more often to prolong my health.

3. Describe how you would like to spend your days after you reach age 100.

I would like to spend my days surrounded by friends and family, spending time with them.

4. What goals would you have for the remaining years of your life if you knew you would live to be 120 ? 
I would want to succeed academically to make sure that I have a bright future and am financially stable at age 120.

\section{Example responses from an older adult in the expansive time horizon condition:}

1. How would this [knowing you will live to 120] change your spending or saving?

I would use a higher percentage of income for saving goals. I would perhaps do remodeling of my house for possible disabilities. I would develop more activities or interests for a longer retirement time.

2. How would this [knowing you will live to 120] change what activities you spend time on?

Would develop more volunteer activities. Would develop skills for a "second career" following retirement.

3. Describe how you would like to spend your days after you reach age 100.

Reading, volunteering, traveling, exercise. Lunches with friends.

4. What goals would you have for the remaining years of your life if you knew you would live to be 120 ?

Be financially independent. Volunteer more. Take classes at University. Travel. Develop second career.

Limited time horizon condition instructions: "People can never know when life will end. For instance, you could die of a sudden heart attack or stroke or in a car accident at any time. Yet much research shows that we spend too little time focusing on the present moment. As you answer the following questions, please plan for a future in which you only live for 6 more months. Assume you will be in good health."

\section{Example responses from a younger adult in the limited time horizon condition:}

1. How would this [knowing you have 6 months left to live] change your spending or saving?

I would not be as frugal as I used to be. I would spend generously on everything and anything I need and want. I would probably spend most of my savings on me and my loved ones.

2. How would this [knowing you have 6 months left to live] change what activities you spend time on?

I would prioritize my activities to not waste any more time. I'd live for the present, doing things that bring more immediate pleasure and joy. I'd spend time with my loved ones, try things I've always wanted to do, and focus on my religious faith.

3. Describe how you would like to spend your last day of life.

My ideal last day of life would be filled with lots of happiness and laughter with my loved ones, whatever it is we are doing. I'd also be practicing my religious faith diligently.

4. What goals would you have for the remaining months of your life if you knew you had only 6 more months to live?

- I would want to make sure I spend more quality time with the people that matter.

- Give more than receive

- Make others smile

- Practice religion
Example responses from an older adult in the limited time horizon condition:

1. How would this [knowing you have 6 months left to live] change your spending or saving?

I would spend more freely, be more indulgent, and fret less about stretching my money.

2. How would this [knowing you have 6 months left to live] change what activities you spend time on?

I would travel more, visit friends and family and 'bucket list' places, and devote more time to hobbies in lieu of work.

3. Describe how you would like to spend your last day of life.

Surrounded by friends and family, smiles, laughing, sharing any hidden or forgotten thoughts... Hugs all around! A good meal with all.

4. What goals would you have for the remaining months of your life if you knew you had only 6 more months to live?

Catalogue my photos, scanning the rest. Drive a Ferrari! Make some progress on my boat model. Donate my time to helping young people and trying to spark their interest in science.

Appendix 2 Full instructions and writing prompt questions, along with two example responses from the control condition of Experiment 2.

\section{Example responses from a participant in the control condition:}

1. What was the most recent thing that you spent money on? This could be a bill that you paid or a purchase that you made.

I just bought a family community agriculture shares (CSAs) for this season.

2. What activities have you completed today?

Worked, walked, did some light shopping.

3. Describe what you plan to do immediately after completing this survey.

Take a walk.

4. What is one activity that you complete every day?

Wake up, take a shower, eat, read a book, check email

\section{Example responses from a participant in the control condition:}

1. What was the most recent thing that you spent money on? This could be a bill that you paid or a purchase that you made.

Shoes

2. What activities have you completed today? Lunch and laundry

3. Describe what you plan to do immediately after completing this survey.

Write music

4. What is one activity that you complete every day? Exercise 


\section{References}

Baumeister, R. F., Bratlavsky, E., Finkenauer, C., \& Vohs, K. D. (2001). Bad is stronger than good. Review of General Psychology, 54(4), 323-370.

Buhrmester, M., Kwang, T., \& Gosling, S. D. (2011). Amazon's Mechanical Turk: A new source of inexpensive, yet high-quality, data? Perspectives on Psychological Science, 6, 3-5.

Cacioppo, J. T., Berntson, G. G., Bechara, A., Tranel, D., \& Hawkley, L. C. (2011). Could an aging brain contribute to subjective well-being? The value added by a social neuroscience perspective. Social Neuroscience: Toward Understanding the Underpinnings of the Social Mind, 249-262.

Carstensen, L. L. (1993). Motivation for social context across the lifespan: A theory of socioemotional selectivity. In J. Jacobs (Ed.), Nebraska symposium on motivation: Developmental perspectives on motivation (Vol. 40, pp. 209-254). Lincoln: University of Nebraska Press.

Carstensen, L. L. (1995). Evidence for a life-span theory of socioemotional selectivity. Current Directions in Psychological Science, 4(5), 151-156.

Carstensen, L. L. (2006). The influence of a sense of time on human development. Science, 312(5782), 1913-1915.

Carstensen, L. L., \& Fredrickson, B. L. (1998). Influence of HIV status and age on cognitive representations of others. Health Psychology, 17(6), 494-503.

Carstensen, L. L., Isaacowitz, D. M., \& Charles, S. T. (1999). Taking time seriously: A theory of socioemotional selectivity. American Psychologist, 54, 165-181.

Charles, S. T., Mather, M., \& Carstensen, L. L. (2003). Aging and emotional memory: The forgettable nature of negative images for older adults. Journal of Experimental Psychology: General, 132(2), 310-324.

Demeyer, I., \& De Raedt, R. (2013). Attentional bias for emotional information in older adults: The role of emotion and future time perspective. PLoS One, 8(6), e65429.

Demeyer, I., \& De Raedt, R. (2014). The effect of future time perspective manipulation on affect and attentional bias. Cognitive Therapy and Research, 38(3), 302-312.

DeWall, C. N., \& Baumeister, R. F. (2007). From terror to joy: Automatic tuning to positive affective information following mortality salience. Psychological Science, 18(11), 984-990.

Fredrickson, B. L. (1995). Socioemotional behavior at the end of college life. Journal of Social and Personal Relationships, 12(2), 261-276.

Fung, H. H., \& Carstensen, L. L. (2003). Sending memorable messages to the old: Age differences in preferences and memory for advertisements. Journal of Personality and Social Psychology, 85(1), 163-178.

Fung, H. H., \& Carstensen, L. L. (2006). Goals change when life's fragility is primed: Lessons learned from older adults, the September 11 attacks and SARS. Social Cognition, 24(3), 248-278.

Fung, H. H., Carstensen, L. L., \& Lutz, A. M. (1999). Influence of time on social preferences: Implications for life-span development. Psychology and Aging, 14(4), 595-604.

Gruhn, D., Smith, J., \& Baltes, P. B. (2005). No aging bias favoring memory for positive material: Evidence from a heterogeneityhomogeneity list paradigm using emotionally toned words. Psychology and Aging, 20, 579-588.

Isaacowitz, D. M., Allard, E. S., Murphy, N. A., \& Schlangel, M. (2009). The time course of age-related preferences toward positive and negative stimuli. The Journals of Gerontology, Series B: Psychological Sciences, 64(2), 188-192. doi:10.1093/geronb/gbn036

Isaacowitz, D. M., Toner, K., Goren, D., \& Wilson, H. R. (2008). Looking while unhappy: Mood congruent gaze in younger adults, positive gaze in older adults. Psychological Science, 19, 848-853.

Isaacowitz, D. M., Toner, K., \& Neupert, S. D. (2009). Use of gaze for real-time mood regulation: Effects of age and attentional functioning. Psychology and Aging, 24, 989-994.

Isaacowitz, D. M., Wadlinger, H. A., Goren, D., \& Wilson, H. R. (2006a). Is there an age-related positivity effect in visual attention? A comparison of two methodologies. Emotion, 6(3), 511-516. doi: $10.1037 / 1528-3542.6 .3 .511$

Isaacowitz, D. M., Wadlinger, H. A., Goren, D., \& Wilson, H. R. (2006b). Selective preference in visual fixation away from negative images in old age? An eye-tracking study. Psychology and Aging, 21(1), 40-48. doi:10.1037/08827974.21.1.40

Kellough, J. L., \& Knight, B. G. (2012). Positivity effects in older adults' perception of facial emotion: The role of future time perspective. The Journals of Gerontology Series B: Psychological Sciences and Social Sciences, 67B(2), 150-158.

Kennedy, Q., Mather, M., \& Carstensen, L. L. (2004). The role of motivation in the age-related positivity effect in autobiographical memory. Psychological Science, 15(3), 208-214.

Kensinger, E. A., Brierley, B., Medford, N., Growdon, J. H., \& Corkin, S. (2002). Effects of normal aging and Alzheimer's disease on emotional memory. Emotion, 2, 118-134.

Knight, M., Seymour, T. L., Gaunt, J. T., Baker, C., Nesmith, K., \& Mather, M. (2007). Aging and goal-directed emotional attention: Distraction reverses emotional biases. Emotion, 7(4), 705-714. doi:10.1037/1528-3542.7.4.705

Kryla-Lighthall, N. R., \& Mather, M. (2009). The role of cognitive control in older adults' emotional well-being. In V. Berngtson, D. Gans, N. Putney, \& M. Silverstein (Eds.), Handbook of theories of aging (2nd ed., pp. 323-344). Springer Publishing.

Labouvie-Vief, G. (2003). Dynamic integration affect, cognition, and the self in adulthood. Current Directions in Psychological Science, 12(6), 201-206.

Labouvie-Vief, G. (2009). Dynamic integration theory: Emotion, cognition, and equilibrium in later life. In V. Bengston, D. Gans, N. M. Pulney, \& M. Silverstein (Eds.), Handbook of theories of aging (2nd ed., Vol. xxv). New York, NY: Springer Publishing Co.

Labouvie-Vief, G., Diehl, M., Jain, E., \& Zhang, F. (2007). Six-year change in affect optimization and affect complexity across the adult life span: A further examination. Psychology and Aging, 22, 738751.

Labouvie-Vief, G., \& Medler, M. (2002). Affect optimization and affect complexity: Modes and styles of regulation in adulthood. Psychology and Aging, 17, 571-588.

Lang, P. J., Bradley, M. M., \& Cuthbert, B. N. (1999). International affective picture system (IAPS): Instruction manual and affective ratings. The center for research in psychophysiology, University of Florida.

Levine, L. J., \& Bluck, S. (1997). Experienced and remembered emotional intensity in older adults. Psychology and Aging, $12(3), 514-523$.

Mather, M. (2016). The affective neuroscience of aging. Annual Review of Psychology, 67, 213-238.

Mather, M., \& Carstensen, L. L. (2005). Aging and motivated cognition: The positivity effect in attention and memory. Trends in Cognitive Science, 9(10), 496-502. doi:10.1016/j. tics.2005.08.005

Mather, M., \& Johnson, M. K. (2000). Choice-supportive source monitoring: Do our decisions seem better to us as we age? Psychology and Aging, 15(4), 596-606.

Mather, M., \& Knight, M. (2005). Goal-directed memory: The role of cognitive control in older adults' emotional memory. Psychology and Aging, 20(4), 554-570.

Nashiro, K., Sakaki, M., \& Mather, M. (2012). Age differences in brain activity during emotion processing: Reflections of agerelated decline or increased emotion regulation? Gerontology, $58,156-163$.

O'Brien, E., \& Ellsworth, P. C. (2012). Saving the last for best: A positivity bias for end experiences. Psychological Science, 23, 163-165. 
Paolacci, G., Chandler, J., \& Ipeirotis, P. G. (2010). Running experiments on amazon mechanical turk. Judgement and Decision Making, 5, 411-419.

Pruzan, K., \& Isaacowitz, D. M. (2006). An attentional application of socioemotional selectivity theory in college students. Social Development, 15(2), 326-338.

Reed, A. E., \& Carstensen, L. L. (2012). The theory behind the agerelated positivity effect. Frontiers in Psychology, 3.
Reed, A. E., Chan, L., \& Mikels, J. A. (2014). Meta-analysis of the agerelated positivity effect: Age differences in preferences for positive over negative information. Psychology and Aging, 29(1), 1-15. doi: $10.1037 / \mathrm{a} 0035194$

Watson, D., Clark, L. A., \& Tellegen, A. (1988). Development and validation of brief measures of positive and negative affect: The PANAS scales. Journal of Personality and Social Psychology, 54, 1063-1070. 